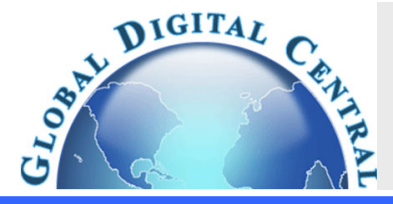

Frontiers in Heat and Mass Transfer

Available at www.ThermalFluidsCentral.org

\title{
LAPLACE TRANSFORM SOLUTION OF UNSTEADY MHD JEFFRY FLUID FLOW PAST VERTICALLY INCLINED PORUS PLATE
}

\author{
K.V. Chandra Sekhar* \\ Koneru Lakshmaiah Education Foundation, Vaddeswaram, Guntur, Andhra Pradesh, India
}

\begin{abstract}
The behavior of unsteady MHD flow of Jeffrey fluid over an inclined porous plate was analyzed in the present article. The governing partial differential equations of the flow phenomena were solved by using powerful mathematical tool Laplace transforms. The variations of velocity, temperature of the flow with respect to dissimilar physical parameters are analyzed through graphs. The parameters of engineering interest are skin friction and Nusselt number. For better understanding of the problem, variations of skin friction and Nusselt number with respect to critical parameters are tabulated.
\end{abstract}

Keywords: Laplace Transform, MHD, Jeffry fluid, Unsteady, Inclined, Porous plate

\section{INTRODUCTION}

Non - Newtonian fluids got good attention by several researchers because of their significant industrial and technical applications. A good number of people have done work on MHD flows. Vijaya $\mathrm{N}$ et al.(2018) thermo physical properties of a Casson fluid through an oscillating vertical wall embedded through porous medium. Vedavathi $\mathrm{N}$ et al.(2017) investigated the radiation effects, of MHD free convective chemically reacting nano fluid over a semi infinite vertical plate. An analytical investigation has been done by Dharmaiah $G$ et al.(2018), for an unsteady, two-dimensional, laminar, boundary layer flow of a viscous incompressible electrically conducting fluid along a semi-infinite vertical permeable moving plate in the presence of Diffusion-thermo and radiation absorption effects. Heat transfer analysis on a stagnation point boundary layer flow of a Jeffrey fluid over a stretching surface was done by Ehtsham A et al.(2019). Dhanalakshmi M et al.(2019) the soret and dufour effect of Kuvshinshiki fluid over MHD free convection flow past a vertical porous plate with heat and mass exchange in companionship of chemical reaction. Kallepalli N.S et al. (2019) studied the behaviour of MHD flow in porous channel under the influence of different critical parameters. Sivaiah G et al. (2019) made a survey of MHD boundary layer flow visco elastic and dissipative fluid past porous plate. Vijaya K et al. (2019) talked about MHD casson fluid flow in presence of radiation soret and chemical reaction effects. Sujatha T et al.(2019) perceived the chemical reaction effect on MHD nanofluid flow over cone and wedge. Ibrahim S.M et al. (2017) investigated heat and mass transfer in a chemically reacting laminar mixed convection flow from a vertical sheet with induced magnetic field. Reddy G.V.R et al. (2018) dicussed the the Soret and Dufour effects on MHD micropolar liquid flow over linearly stretching sheet. Jayaramireddy $\mathrm{K}$ et al.(2018) investigated MHD chemically reacting Casson nano fluid flow over non linear permeable strtching sheet in presence of thermal radiation, viscous dissipation. Naga santoshi $\mathrm{P}$ et al.(2018) have done analysis on heat and mass transfer of mixed convection MHD Casson Nano fluid flow over a stretching sheet with variable viscosity and non-uniform heat source. Charan kumar $\mathrm{G}$ et al.(2018) described the effects of Joule heating and chemical reaction on unsteady MHD mixed convective micro polar fluid over a stretching sheet in presence of radiation, non-uniform heat source and porous medium. Krishna Y.H et al.(2018) addressed the magneto hydrodynamic flow of a Casson fluid over a permeable stretching sheet in the presence of mass transfer From above survey in view of their differences with Newtonian fluids, several models of nonNewtonian fluids have been proposed. The familiar and simple model of non- Newtonian fluid is Jeffrey fluid. Sunitha Rani Y et al.(2019) presented the finite element analysis of MHD Jeffrey fluid over inclined plate. As an extension in the present analysis MHD flow of Jeffrey fluid above slanted vertical permeable plate was considered. The governing equations are solved to get the exact analytical solution applying Laplace transform technique. The results are in good agreement with Sunitha Rani Y et al.[2019].

\section{ANALYSIS OF THE PROBLEM}

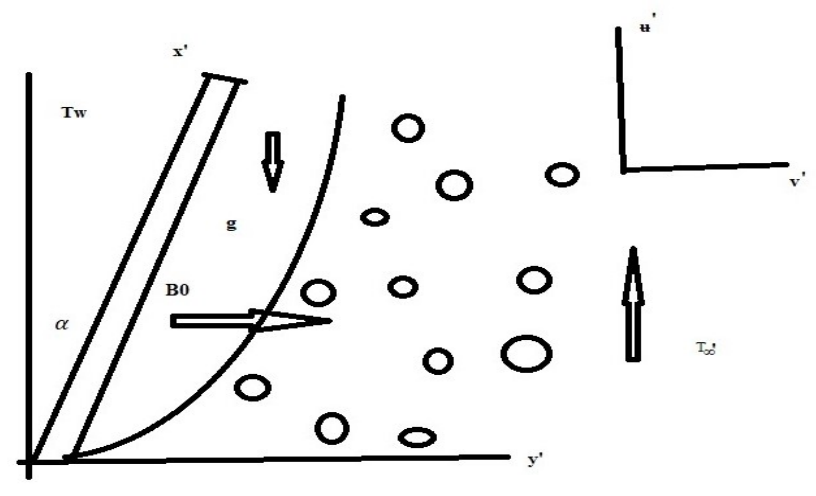

Fig. 1 Geometry of the problem

The plate is taken along $\mathrm{x}^{\prime}$ - direction and $\mathrm{y}^{\prime}$ is taken normal to the plate. The plate is considered of infinite length along $\mathrm{X}^{\prime}$ direction and so all the physical parameters will not depend on $\mathrm{X}^{\prime}$. The velocity components along $\mathrm{x}^{\prime}$ and $\mathrm{y}^{\prime}$ direction are taken as $\mathrm{u}^{\prime}$ and $\mathrm{v}^{\prime}$ 
respectively. $\quad \mathrm{T}_{\mathrm{W}}^{\prime}$ and $\mathrm{T}_{\infty}^{\prime}$ are the temperatures at wall and free stream temperatures. A uniform magnetic field of strength $\mathrm{B}_{0}$ is applied normal to the plate. The transverse magnetic field and magnetic Reynolds number are assumed to be very small so that the produced magnetic field is negligible. An unsteady MHD free convective, incompressible, electrically conducting fluid flow over an inclined permeable plate was considered. The equations narrating the flow are given as follows.

$\frac{\partial \mathrm{v}^{\prime}}{\partial \mathrm{y}^{\prime}}=0 \quad\left(\mathrm{v}^{\prime}\right.$ is cons tant $)$

$\frac{\partial \mathrm{u}^{\prime}}{\partial \mathrm{t}^{\prime}}+\mathrm{v}^{\prime} \frac{\partial \mathrm{u}^{\prime}}{\partial \mathrm{y}^{\prime}}=\mathrm{g} \beta\left(\mathrm{T}^{\prime}-\mathrm{T}_{\infty}^{\prime}\right) \cos \alpha$

$+\left(\frac{v}{1+\lambda}\right) \frac{\partial^{2} \mathrm{u}^{\prime}}{\partial \mathrm{y}^{\prime 2}}-\frac{\sigma \mathrm{B}_{0}^{2} \mathrm{u}^{\prime}}{\rho}-\frac{v \mathrm{u}^{\prime}}{\mathrm{k}^{\prime}}$

$\frac{\partial \mathrm{T}^{\prime}}{\partial \mathrm{t}^{\prime}}+\mathrm{v}^{\prime} \frac{\partial \mathrm{T}^{\prime}}{\partial \mathrm{y}^{\prime}}=\frac{\kappa}{\rho \mathrm{c}_{\mathrm{p}}} \frac{\partial^{2} \mathrm{~T}^{\prime}}{\partial \mathrm{y}^{\prime 2}}$

The associated boundary conditions of the flow are

$$
\begin{aligned}
& \mathrm{u}^{\prime}=0, \mathrm{v}^{\prime}=-\mathrm{v}_{0}, \mathrm{~T}^{\prime}=\mathrm{T}_{\mathrm{W}}^{\prime}+\varepsilon\left(\mathrm{T}_{\mathrm{W}}^{\prime}-\mathrm{T}_{\infty}^{\prime}\right) \mathrm{e}^{\mathrm{i} \omega^{\prime} \mathrm{t}^{\prime}} \text { at } \mathrm{y}^{\prime}=0 \\
& \mathrm{u}^{\prime} \rightarrow 0, \mathrm{~T}^{\prime} \rightarrow \mathrm{T}_{\infty}^{\prime} \text { as } \mathrm{y}^{\prime} \rightarrow \infty
\end{aligned}
$$

The non dimensional variables and parameters are

$$
\begin{aligned}
& \mathrm{y}=\frac{\mathrm{y}^{\prime} v_{0}}{v}, \mathrm{t}=\frac{\mathrm{t}^{\prime} v_{0}^{2}}{4 v}, \omega=\frac{4 v \omega^{\prime}}{v_{0}{ }^{2}}, \mathrm{u}=\frac{\mathrm{u}^{\prime}}{v_{0}}, \mathrm{M}=\frac{\sigma \mathrm{B}_{0}^{2} v}{\rho v_{0}{ }^{2}} \\
& \theta=\frac{\mathrm{T}^{\prime}-\mathrm{T}^{\prime} \infty}{\mathrm{T}_{\mathrm{W}}^{\prime}-\mathrm{T}_{\infty}^{\prime}}, \operatorname{Pr}=\frac{v \rho \mathrm{c}_{\mathrm{p}}}{\kappa}, \mathrm{Gr}=\frac{v g \beta\left(\mathrm{T}_{\mathrm{W}}^{\prime}-\mathrm{T}^{\prime}\right)_{\infty}}{v_{0} 3}, \mathrm{k}=\frac{\mathrm{k}^{\prime} v_{0}{ }^{2}}{v^{2}}
\end{aligned}
$$

Using the (6) in equations (2) and (3)

$$
\begin{aligned}
& \frac{1}{4} \frac{\partial \mathrm{u}}{\partial \mathrm{t}}-\frac{\partial \mathrm{u}}{\partial \mathrm{y}}=\operatorname{Gr} \cos \alpha \theta+\left(\frac{1}{1+\lambda}\right) \frac{\partial^{2} \mathrm{u}}{\partial \mathrm{y}^{2}}-\left(\mathrm{M}+\frac{1}{\mathrm{k}}\right) \\
& \frac{1}{4} \frac{\partial \theta}{\partial \mathrm{t}}-\frac{\partial \theta}{\partial \mathrm{y}}=\frac{1}{\operatorname{Pr}} \frac{\partial^{2} \theta}{\partial \mathrm{y}^{2}}
\end{aligned}
$$

The transformed boundary conditions are

$$
\begin{aligned}
& \mathrm{u}=0, \theta=1+\varepsilon \mathrm{e}^{\mathrm{i} \omega \mathrm{t}} \text { at } \mathrm{y}=0 \\
& \mathrm{u} \rightarrow 0, \theta \rightarrow 0 \text { as } \mathrm{y} \rightarrow \infty
\end{aligned}
$$

\section{Laplace Transform Solution}

Laplace transform technique was employed to get exact solution of the problem. Applying Laplace transform for the equations (7) and (8) becomes ordinary differential equations of second order.

$$
\begin{aligned}
& \frac{d^{2} u}{d y^{2}}-D \frac{d u}{d y}-(e-s f) u=G \cos (\alpha) \theta(y, s) \\
& \frac{d^{2} \theta}{d y^{2}}+\operatorname{Pr} \frac{d \theta}{d y}-\frac{\operatorname{Pr} s}{4} \theta=-\frac{\operatorname{Pr}}{4} \theta(y, 0)
\end{aligned}
$$

and the boundary conditions (9) and (10) becomes

$$
\mathrm{u}(\mathrm{y}, \mathrm{s})=0, \theta(\mathrm{y}, \mathrm{s})=\frac{1}{\mathrm{~s}}+\varepsilon \frac{1}{\mathrm{~s}-\mathrm{i} \omega} \text { at } \mathrm{y}=0
$$

$\mathrm{u}(\mathrm{y}, \mathrm{s}) \rightarrow 0, \theta(\mathrm{y}, \mathrm{s}) \rightarrow 0$ as $\mathrm{y} \rightarrow \infty$

Solving the equations (11) and (12) along with the boundary conditions we obtain the expressions for the solutions of (11) and (12) as

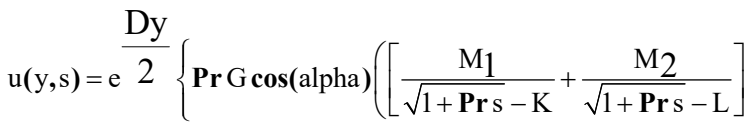

$$
\begin{aligned}
& +\varepsilon\left[\frac{\mathrm{M}_{5}}{\sqrt{1+\operatorname{Prs}}-\mathrm{K}}+\frac{\mathrm{M}_{6}}{\sqrt{1+\operatorname{Prs}}-\mathrm{L}}\right) \\
& \left.\left.+\left(\frac{\mathrm{M} 7}{\sqrt{1+\operatorname{Prs}}-\mathrm{N}_{4}}-\frac{\mathrm{M}_{7}}{\sqrt{1+\operatorname{Pr} \mathrm{s}}+\mathrm{N}_{4}}\right]\right)\right\}
\end{aligned}
$$

$$
\begin{aligned}
& \theta(\mathrm{y}, \mathrm{s})= \\
& \mathrm{e}^{\frac{-\mathrm{y} \mathbf{P r}}{2}}\left(\frac{1-\mathrm{k}}{\mathrm{s}}+\varepsilon \frac{1}{\mathrm{~s}-\mathrm{i} \omega}\right) \mathrm{e}^{\frac{-\mathrm{yPr} \sqrt{1+\operatorname{Pr} \mathrm{s}}}{2}}+\frac{\mathrm{k}}{\mathrm{s}} \mathrm{e}^{-\mathrm{y} \operatorname{Pr} \sqrt{1+\operatorname{Pr} \mathrm{s}}}
\end{aligned}
$$

Applying inverse Laplace transform for equations (15) and (16) we get the expressions for velocity and temperature distributions of the flow as follows

$$
\begin{aligned}
& u(y, t)=e^{\frac{D y}{2}} \operatorname{Pr} G \cos \alpha\left[2 K_{1} e^{-y \sqrt{N_{1}}+K^{2} t}\right. \\
& \left.+2 \mathrm{LM}_{2} \mathrm{e}^{-\mathrm{y} \sqrt{\mathrm{N}_{1}}+\mathrm{L}^{2} \mathrm{t}}\right]-\varepsilon\left[2 \mathrm{KM}_{5} \mathrm{e}^{-\mathrm{y} \sqrt{\mathrm{N}_{1}}+\mathrm{L}^{2} \mathrm{t}}\right. \\
& +2 \mathrm{LM}_{6} \mathrm{e}^{-\mathrm{y} \sqrt{\mathrm{N}_{2}}+\mathrm{L}^{2} \mathrm{t}}+2 \mathrm{~N}_{4} \mathrm{M}_{7} \mathrm{e}^{\left.-\mathrm{y} \sqrt{\mathrm{N}_{3}}+\mathrm{N}_{4}{ }^{2} \mathrm{t}\right]} \\
& +\operatorname{Pr} G \cos \alpha e e^{\frac{-y}{2}}\left[M_{1}\left(\frac{1}{\sqrt{\pi t}} e^{\frac{-y^{2} \mathbf{P r}}{16 t}}+K e^{\left(K^{2} t-K y\right)} \operatorname{erfc}\left(\frac{y \sqrt{P r}}{4 \sqrt{t}}-K \sqrt{t}\right)\right)\right.
\end{aligned}
$$

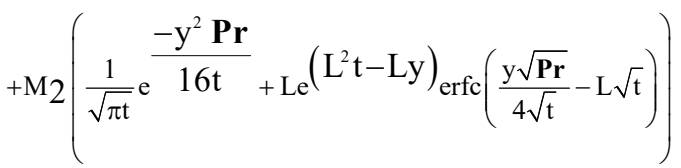

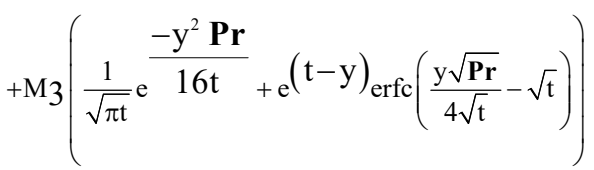

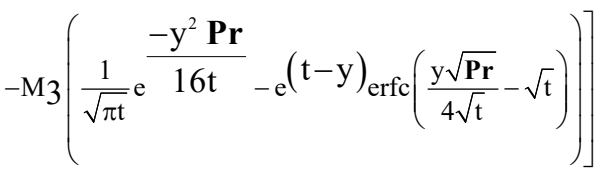

$$
\begin{aligned}
& +\varepsilon \frac{-y \mathbf{P r}}{2}\left[\mathrm{M}_{5}\left(\frac{1}{\sqrt{\pi \mathrm{t}}} \mathrm{e}^{\frac{-\mathrm{y}^{2} \mathbf{P r}}{16 \mathrm{t}}+\mathrm{Ke}}\left(\mathrm{K}^{2} \mathrm{t}-\mathrm{Ky}\right)_{\operatorname{erfc}}\left(\frac{\mathrm{y} \sqrt{\mathbf{P r}}}{4 \sqrt{\mathrm{t}}}-\mathbf{K} \sqrt{\mathrm{t}}\right)\right)\right. \\
& +\mathrm{M}_{6}\left(\frac{1}{\sqrt{\pi \mathrm{t}}} \mathrm{e}^{\frac{-\mathrm{y}^{2} \mathbf{P r}}{16 \mathrm{t}}}+\mathrm{Le} \mathrm{L}^{\left.\left(\mathrm{L}^{2} \mathrm{t}-\mathrm{Ly}\right)_{\operatorname{erfc}}\left(\frac{\mathrm{y} \sqrt{\mathbf{P r}}}{4 \sqrt{\mathrm{t}}}-\mathrm{L} \sqrt{\mathrm{t}}\right)\right)}\right.
\end{aligned}
$$




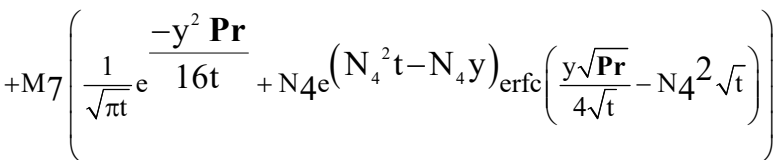

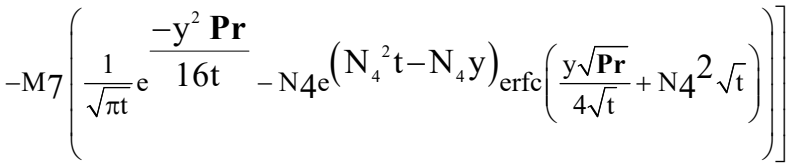

$$
\begin{aligned}
& \theta(y, t)=e^{-b}\left[\frac{\operatorname{Pr}}{2}\left\{\begin{array}{l}
e^{-b+t} \operatorname{erfc}\left(\frac{b}{2 \sqrt{t}}-\sqrt{t}\right) \\
+e^{b+t} \operatorname{erfc}\left(\frac{b}{2 \sqrt{t}}+\sqrt{t}\right)
\end{array}\right\}\right] \\
& +\varepsilon e^{-b}\left[\frac{\operatorname{Pr}}{2}\left\{\begin{array}{l}
e^{-b N_{4}+N_{4}{ }^{2} t} \operatorname{erfc}\left(\frac{b}{2 \sqrt{t}}-N_{4} \sqrt{t}\right) \\
\left.+e^{b+t_{e r f c}\left(\frac{b}{2 \sqrt{t}}+N_{4} \sqrt{t}\right.}\right)
\end{array}\right\}\right]
\end{aligned}
$$

\section{SKIN FRICTION AND RATE OF HEAT TRANSFER}

The physical parameters of engineering interest are skin friction and Nusselt number. These parameters are useful to narrate the fluid flow and exchange of temperature near to the plate. The non dimensional skin friction and Nusselt number are

$$
\begin{aligned}
& \mathrm{sf}=-\left(\frac{\partial u}{\partial y}\right)_{y=0} \\
& \mathrm{Nu}=-\left(\frac{\partial \theta}{\partial y}\right)_{y=0}
\end{aligned}
$$

\section{RESULTS AND DISCUSSIONS}

The equations narrating the flow are resolved by employing Laplace transform method, to get exact analytical solutions for velocity and temperature. The influence of various physical parameters on fluid flow were analyzed graphically. The variations in skin friction, Nusselt number with respect to different physical parameters were illustrated through tables. The range of the variable $\mathrm{y}$ was taken in between 0 and 6. It is observed that the profiles of velocity and temperature fields reflecting the boundary conditions of the problem.

Figure -2 displays the velocity profiles for different values of inclined angle. It reflects the rise in angle of inclination slow down the velocity of the fluid, because of buoyancy force due to gravity. Figure -3 shows that increase of Jeffry fluid parameter increases fluid velocity. Figure 4 depicts increase of thermal grashof number enhances fluid velocity. This is due to the fact increment of grashof number increases the buoyancy forces that lead to rise in liquid velocity. Figure -5 shows the impact of permeability parameter on fluid flow. This is because porous medium opposes the fluid flow. Lorentz forces resist the fluid flow which was accompanied with magnetic parameter. Therefore increase of magnetic parameter declines the fluid velocity. This was depicted in figure -6 . Figure -7 shows the impact of Prandtl number on velocity profiles. Increase of Prandtl number results in decrease in thermal conductivity of the fluid, and hence temperature of the fluid decreases.

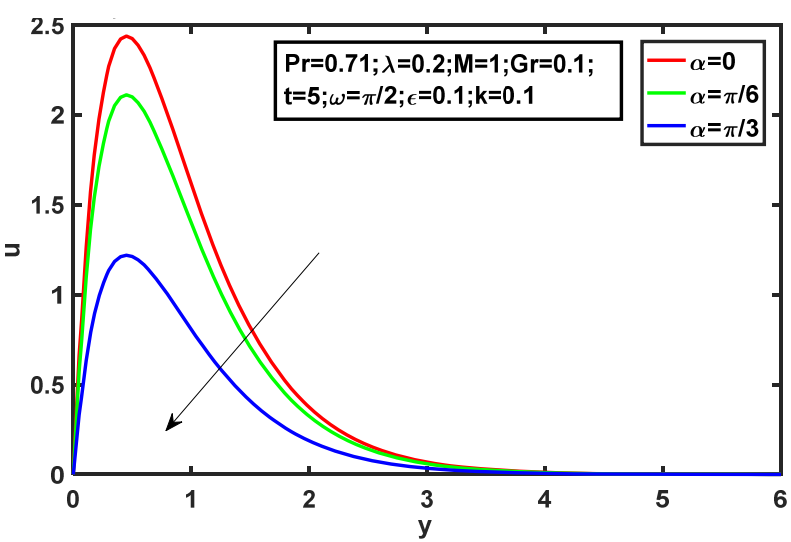

Fig. 2 Variation of velocity for dissimilar values of $\alpha$

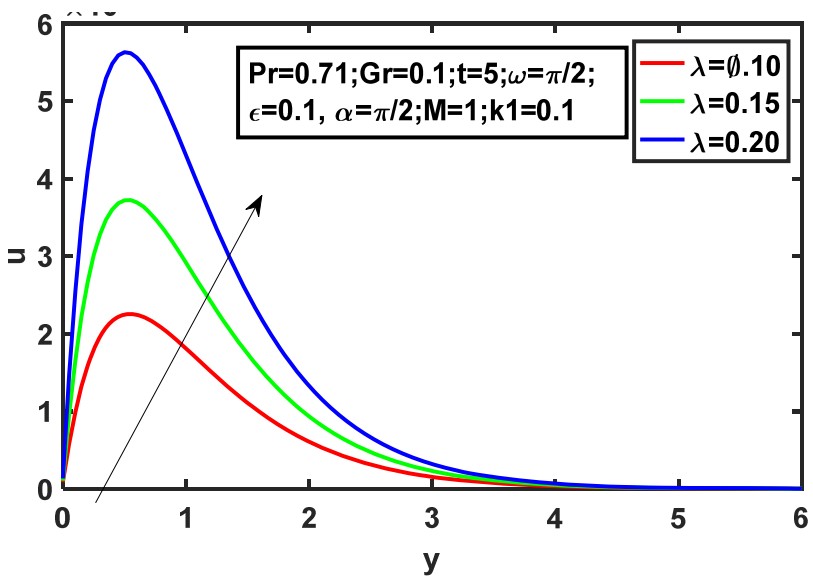

Fig. 3 Variation of velocity for dissimilar values of $\lambda$

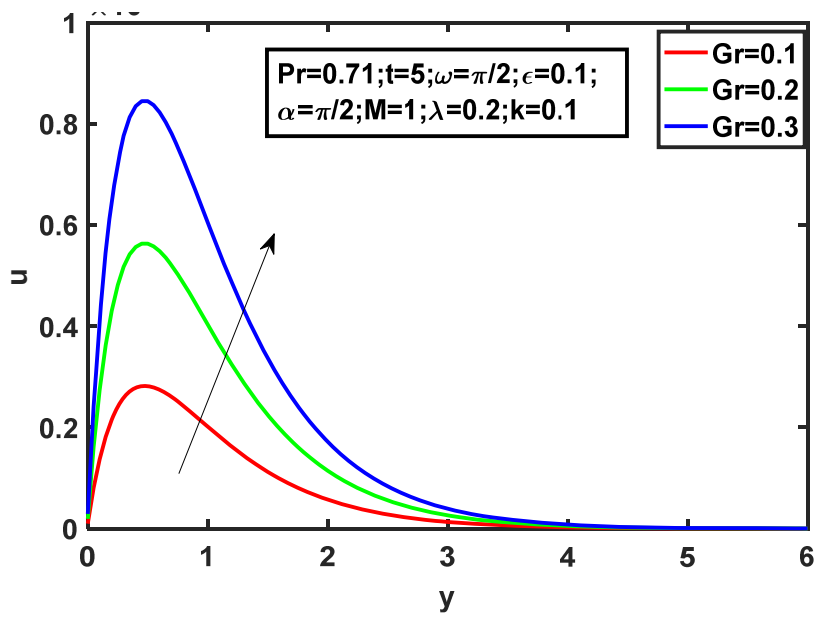

Fig. 4 Variation of velocity for dissimilar values of $\mathrm{Gr}$ 


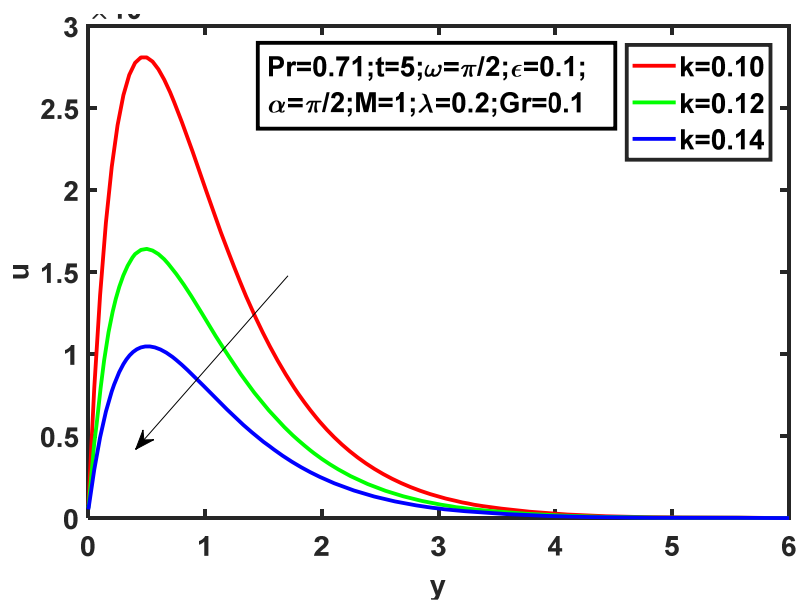

Fig. 5 Variation of velocity for dissimilar values of $k$

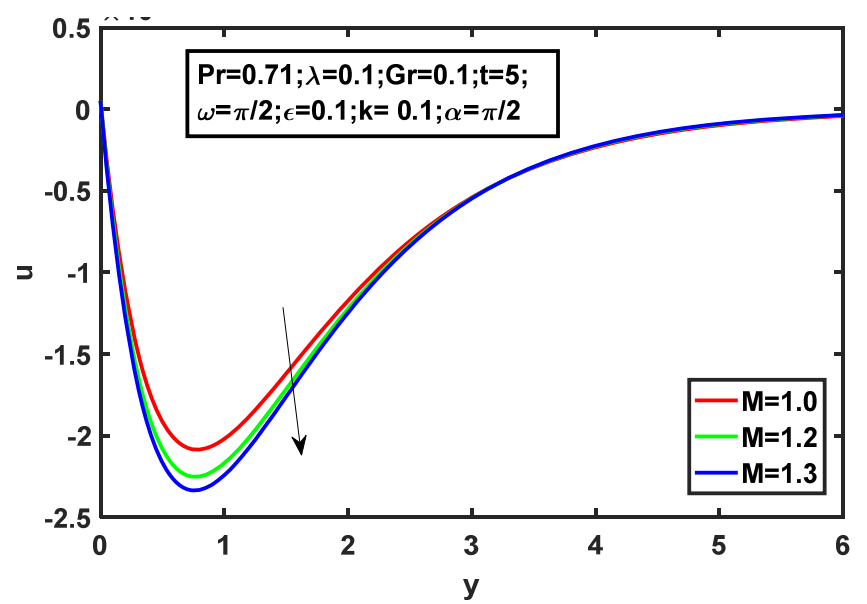

Fig. 6 Variation of velocity for dissimilar values of $M$

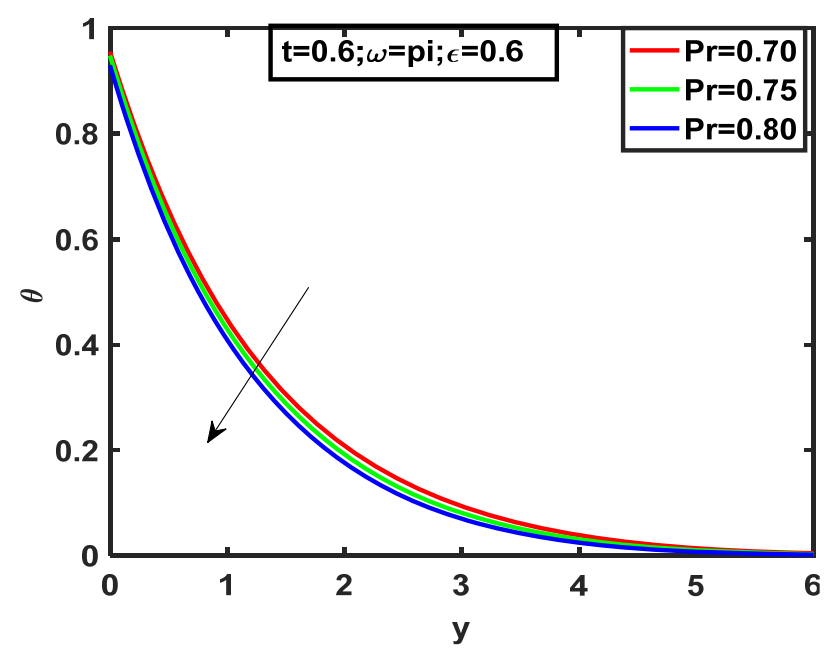

Fig. 7 Variation of temperature for dissimilar values of $P r$

Tables 1 and 2 analyze the change in skin friction and Nusselt number under the influence of different physical parameters.
Table 1 Variation of skin friction for different physical parameters

\begin{tabular}{|c|c|c|c|c|c|c|}
\hline $\mathrm{Gr}$ & $\mathrm{k}$ & $\mathrm{M}$ & $\mathrm{Pr}$ & $\alpha$ & $\lambda$ & $\mathrm{sf}$ \\
\hline 0.1 & 0.1 & 1 & 0.71 & 0 & 0.1 & 1.6396 \\
\hline 0.2 & 0.1 & 1 & 0.71 & 0 & 0.1 & 3.2793 \\
\hline 0.3 & 0.1 & 1 & 0.71 & 0 & 0.1 & 4.9190 \\
\hline 0.1 & 0.1 & 1 & 0.71 & 0 & 0.1 & 1.6396 \\
\hline 0.1 & 0.2 & 1 & 0.71 & 0 & 0.1 & -0.7401 \\
\hline 0.1 & 0.3 & 1 & 0.71 & 0 & 0.1 & -0.7821 \\
\hline 0.1 & 0.1 & 1 & 0.71 & 0 & 0.1 & 1.6396 \\
\hline 0.1 & 0.1 & 2 & 0.71 & 0 & 0.1 & 2.8005 \\
\hline 0.1 & 0.1 & 3 & 0.71 & 0 & 0.1 & 4.2873 \\
\hline 0.1 & 0.1 & 1 & 0.71 & 0 & 0.1 & 1.6396 \\
\hline 0.1 & 0.1 & 1 & 4 & 0 & 0.1 & 5.1381 \\
\hline 0.1 & 0.1 & 1 & 7 & 0 & 0.1 & 6.5435 \\
\hline 0.1 & 0.1 & 1 & 0.71 & 0 & 0.1 & 1.6396 \\
\hline 0.1 & 0.1 & 1 & 0.71 & 0 & 0.2 & -0.7401 \\
\hline 0.1 & 0.1 & 1 & 0.71 & 0 & 0.3 & -0.7821 \\
\hline
\end{tabular}

Table 2 Variation of Nusselt number with Prandtl number

\begin{tabular}{|c|c|c|}
\hline Pr & $\mathrm{t}$ & $\mathrm{nu}$ \\
\hline 0.70 & 0.6 & 0.8804 \\
\hline 0.75 & 0.6 & 1.0109 \\
\hline 0.80 & 0.6 & 1.15055 \\
\hline
\end{tabular}

\section{Conclusions}

Analysis of unsteady MHD Jeffry fluid flow was done in the present article. Laplace transform technique was employed for the governing equations of the flow. The governing equations are first transformed into a set of normalized equations and then solved analytically applying Laplace transform technique to obtain the general solution. The expressions for velocity and temperature were obtained exponential and error functions. For better understanding of the problem the outcome of the phenomenon was illustrated graphically to explain the behavior of the fluid flow under the influence of different physical parameters. The velocity profiles are starting at origin and converging to zero far away from the plate. Whereas temperature profiles starting at 1 at origin and far away from the plate coinciding with the axis. This shows that the obtained solution is satisfying the boundary conditions of the present problem. Change in skin friction and Nusselt numbers with respect to different critical parameters was shown with tables. During the analysis the following conclusions were drawn. The present work can be extended by incorporating Newtonian heating for the temperature equation.

1. Angle of inclination $\alpha$ declines the speed the fluid

2. Fluid velocity escalates with rise in Jeffry parameter.

3. Thermal grashof number enhances velocity of the fluid

4. Permeability declines the fluid velocity

5. Magnetic parameter lowers fluid velocity

6. Temperature distribution of the fluid drops down for growing values of Prandtl number.

7. From Table 1, we can observe that increase of Prandtl number and Magnetic parameter raises skin friction.

8. Rise in Jeffry parameter lowers skin friction

9. Increase in permeability parameter lowers skin friction

10. Nusselt number increases for growing values of Prandtl number 


\section{Nomenclature}

\begin{tabular}{ll}
$u^{\prime}$ & Velocity component in $x^{\prime}-$ direction \\
$v^{\prime}$ & Velocity component in $y^{\prime}-$ direction \\
$\mathrm{u}$ & Dimensionless velacity \\
${ }^{\mathrm{c}_{\mathrm{p}}}$ & Specific heat at constant pressure \\
$\mathrm{g}$ & Acceleration due to gravity \\
$T$ & Dimensional temperature \\
$T_{w}$ & Wall dimensional temperature \\
$T_{\infty}$ & Free stream dimensional temperature \\
$\theta$ & Dimensionless temperature \\
$P_{r}$ & Prandtl number \\
$B_{0}$ & Uniform magnetic field \\
$\mathrm{k}$ & Permeability parameter \\
$\mathrm{k}^{\prime}$ & Permeability of the porous media \\
$\mathrm{M}$ & Magnetic parameter \\
$\mathrm{t}^{\prime}$ & Dimensional time \\
$\mathrm{t}$ & Dimensionless time \\
$G r$ & Thermal grashof number \\
\hline
\end{tabular}

Greek symbols

$\begin{array}{ll}v & \text { Kinematic viscosity } \\ \alpha & \text { Angle of inclination } \\ \beta & \text { Volumetric coefficient of thermal expansion } \\ \omega & \text { The constant } \\ \varepsilon & \text { A real positive constant }<1 \\ \lambda & \text { Jeffry parameter } \\ \sigma & \text { Electrical conductivity } \\ \rho & \text { Fluid density }\end{array}$

\section{REFERENCES}

Charan Kumar G., Jayarami Reddy K., Ramakrishna K., Narendradh Reddy M. (2018), "Non-uniform heat source/sink and joule heating effects on chemically radiative MHD mixed convective flow of micropolar fluid over a stretching sheet in porous medium," Defect and Diffusion Forum ,388,281-302. https://doi.org/10.4028/www.scientific.net/DDF.388.281

Dhanalakshmi M., Jyothi V., Jayarami Reddy K. (2019), "Soret and Dufour Effects on MHD Convective Flow Past a Vertical Plate Through Porous Medium," Journal of Physics: Conference Series, 1344(1),1-11.

https://doi.org/10.1088/1742-6596/1344/1/012008

Dharmaiah G., Baby Rani C., Vedavathi N., Balamurugan K.S.(2018), "Heat and mass transfer on MHD fluid flow over a semi infinite flat plate with radiation absorption, heat source and diffusion thermo effect," Frontiers in Heat and Mass Transfer, 11(6), 1-8

http://dx.doi.org/10.5098/hmt.11.6
Ehtsham, A., Iqbal,Z and Maraj, E.N.(2019), "Viscous dissipation performance on stagnation point flow of Jeffrey fluid inspired by internal heat generation and chemical reaction," Thermal Science and Engineering Progress, 13, https://doi.org/10.1016/j.tsep.2019.100377

Ibrahim S.M., Mabood F., Suneetha K., Lorenzini G. (2017), "Effects of chemical reaction on combined heat and mass transfer by laminar mixed convection flow from vertical surface with induced magnetic field and radiation," Journal of Engineering Thermophysics, 26(2), 234-255. https://doi.org/10.1134/S1810232817020084

Kallepalli N.S., Rajasekhar K., Ramana Murthy C.V. (2019), "Influence of critical parameters on an unsteady state mhd flow in a porous channel with exponentially decreasing suction," Journal of Mathematical and Computational Science, 9(6), 764-783. https://doi.org/10.28919/jmcs/4249

Konda J.R., Madhusudhana N.P., Konijeti R.(2018), "MHD mixed convection flow of radiating and chemically reactive Casson nanofluid over a nonlinear permeable stretching sheet with viscous dissipation and heat source," Multidiscipline Modeling in Materials and Structures ,14(3), 609-630 https://doi.org/10.1108/MMMS-10-2017-0127

Krishna Y.H., Reddy G.V.R., Makinde O.D.(2018), “Chemical reaction effect on MHD flow of casson fluid with porous stretching sheet," Defect and Diffusion Forum ,389, 100-109 https://doi.org/10.4028/www.scientific.net/DDF.389.100

Naga santoshi P, Reddy G.V.R, Gnaneswara Reddy M,Padma P.(2018), "Heat and Mass Transfer of Non-Newtonian Nanofluid Flow Over a Stretching Sheet with Non-Uniform Heat Source and Variable Viscosity,"(5),821-832.

https://doi.org/10.1166/jon.2018.1517

Reddy G.V.R., Krishna Y.H.(2018), "Soret and dufour effects on MHD micropolar fluid flow over a linearly stretching sheet, through a non-darcy porous medium," International Journal of Applied Mechanics and Engineering ,23(2), 485-502.

https://doi.org/10.2478/ijame-2018-0028

Sivaiah G., Jayarami Reddy K., Chandra Reddy P., Raju M.C. (2019), "Numerical study of mhd boundary layer flow of a viscoelastic and dissipative fluid past a porous plate in the presence of thermal radiation," International Journal of Fluid Mechanics Research, 46(1), 27-38.

http://dx.doi.org/10.1615/InterJFluidMechRes.2018020153

Sujatha T., Jayarami Reddy K., Kumar J.G. (2019), “Chemical reaction effect on nonlinear radiative MHD nanofluid flow over cone and wedge," Defect and Diffusion Forum, 393, 83-102 https://doi.org/10.4028/www.scientific.net/DDF.393.83

Sunitha Rani Y, Jithender Reddy G, Srinivas Raju R(2019), “ Finite element solutions of Jeffry fluid flow past vertically inclined plate in presence of MHD," AIP Conference proceedings 2142, 170007(2019).

https://doi.org/10.1063/1.5122604

Vedavathi N., Dharmaiah G., Balamurugan K.S., Prakash J. (2017), "Heat transfer on mhd nanofluid flow over a semi infinite flat plate embedded in a porous medium with radiation absorption, heat source and diffusion thermo effect," Frontiers in Heat and Mass Transfer, 9(38), 1-8.

http://dx.doi.org/10.5098/hmt.9.38 
Vijaya K., Reddy G.V.R. (2019), "Magneto hydrodynamic casson fluid flow over a vertical porous plate in the presence of radiation, soret and chemical reaction effects," Journal of Nanofluids, 8(6), $1240-1248$

https://doi.org/10.1166/jon.2019.1684

Vijaya N., Hari Krishna Y., Kalyani K., Reddy G.V.R. (2018), "Soret and radiation effects on an unsteady flow of a casson fluid through porous vertical channel with expansion and contraction,"

Frontiers in Heat and Mass Transfer,11,1-11. http://dx.doi.org/10.5098/hmt.11.19

\section{Appendix}

$$
\begin{gathered}
\mathrm{a}=\operatorname{Pr}, \mathrm{b}=\frac{\mathrm{y} \mathbf{P r}}{2}, \mathrm{c}=\mathrm{y} \operatorname{Pr}, \mathrm{d}=\frac{1}{1+\lambda}, \mathrm{e}=\frac{\mathrm{M}+\frac{1}{\mathrm{k}}}{\frac{1}{1+\lambda}} \\
\mathrm{f}=\frac{1}{4(1+\lambda)}, \mathrm{G}=\frac{\mathrm{Gr}}{1+\lambda}, \mathrm{H}=\mathrm{d}^{2}+4 \mathrm{e},
\end{gathered}
$$

$$
\begin{gathered}
\mathrm{A}=\frac{\mathbf{P r}^{2}}{4}+\frac{\mathrm{f}}{\mathrm{a}}, \mathrm{B}=\frac{\mathbf{P r}^{2}}{2}-\frac{\mathrm{d} \mathbf{P r}}{2}, \mathrm{C}=\frac{\mathbf{P r}^{2}}{4}-\frac{\mathrm{d} \mathbf{P r}}{2}-\left(\frac{\mathrm{a}+\mathrm{f}}{\mathrm{a}}\right) \\
\mathrm{K}=\frac{-\mathrm{B}-\sqrt{\mathrm{B}^{2}-4 \mathrm{AC}}}{2 \mathrm{~A}}, \mathrm{~L}=\frac{-\mathrm{B}+\sqrt{\mathrm{B}^{2}-4 \mathrm{AC}}}{2 \mathrm{~A}} \\
\mathrm{M}_{1}=\frac{1}{(\mathrm{~K}-\mathrm{L})\left(\mathrm{K}^{2}-1\right)}, \mathrm{M}_{2}=\frac{1}{(\mathrm{~L}-\mathrm{K})\left(\mathrm{L}^{2}-1\right)}, \mathrm{M}_{4}=-\mathrm{M}_{3} \\
\mathrm{M}_{5}=\frac{1}{(\mathrm{~K}-\mathrm{L})\left(\mathrm{K}-\mathrm{N}_{4}\right)\left(\mathrm{K}+\mathrm{N}_{4}\right)}, \mathrm{M}_{6}=\frac{1}{(\mathrm{~L}-\mathrm{K})\left(\mathrm{L}-\mathrm{N}_{4}\right)\left(\mathrm{L}+\mathrm{N}_{4}\right)} \\
\mathrm{M}_{7}=\frac{1}{2\left(\mathrm{~N}_{4}-\mathrm{K}\right)\left(\mathrm{N}_{4}-\mathrm{L}\right) \mathrm{N}_{1}}, \mathrm{M}_{8}=-\mathrm{M}_{7} \\
\mathrm{~N}_{1}=\mathrm{H}-\frac{\left(\mathrm{K}^{2}-1\right) \mathrm{f}}{\mathrm{a}}, \mathrm{N}_{2}=\mathrm{H}-\frac{\left(\mathrm{L}^{2}-1\right) \mathrm{f}}{\mathrm{a}}, \mathrm{N}_{3}=\mathrm{H}-\mathrm{i} \omega \mathrm{f}, \\
\mathrm{N}_{4}=\sqrt{1+\mathrm{ai} \omega}
\end{gathered}
$$

